\title{
SECOND EOSCPILOT STAKEHOLDERS FORUM (WIEN, 21.-22. NOVEMBER 2018)
}

\section{von Thomas Haselwanter}

Zusammenfassung: Bericht vom letzten "EOSCpilot Stakeholders Forum", 21. und 22. November 2018 im Austria Center in Wien. Einen Tag vor dem Launch der "European Open Science Cloud" (EOSC) unter österreichischer EU-Ratspräsidentschaft wurde hier vorgestellt, wie weit die Initiativen zur Implementierung dieses ambitionierten Projekts schon gekommen sind. Präsentationen zu Governance und technischer Umsetzung wechselten mit Diskussionen über noch ungeklärte oder unfertige Themen ab und gaben einen Vorgeschmack darauf, wie die ab jetzt "Stakeholders Forum" genannte Veranstaltung zukünftig über die Weiterentwicklung der EOSC mitbestimmen wird.

Schlagwörter: Europäische Union; European Open Science Cloud; EOSC; e-Infrastrukturen; Vienna Declaration on the European Open Science Cloud; Universität Wien; 2018; Tagungsbericht

\section{SECOND EOSCPILOT STAKEHOLDERS FORUM (VIENNA, NOVEMBER 21-22, 2018)}

Abstract: Report from the last „EOSCpilot Stakeholders Forum", $21^{\text {st }}$ and $22^{\text {nd }}$ November 2018 at the Austria Center in Vienna. One day before the launch of the „European Open Science Cloud" (EOSC) under the Austrian EU Council Presiden$c y$, this event presented how far the initiatives for implementing this ambitious project have come. Presentations on governance and technical implementation alternated with discussions on unresolved or unfinished topics and gave a foretaste of how meetings like this, now called "Stakeholders Forum", will in future be instrumental in defining the further development of the EOSC.

Keywords: European Union; European Open Science Cloud; EOSC; e-Infrastructures; Vienna Declaration on the European Open Science Cloud; University of Vienna; 2018; conference report

DOI: https://doi.org/10.31263/voebm.v72i1.2285

(C) Thomas Haselwanter

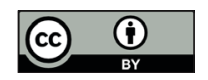

Dieses Werk ist lizenziert unter einer

Creative-Commons-Lizenz Namensnennung 4.0 International 


\section{Gemeinsam forschen in der EOSC}

Juan Bicarregui (Koordinator EOSCpilot) erklärte in seiner Einleitung die Aufgaben des Stakeholder Forums ${ }^{1}$, das - offen für alle Stakeholder, die mit Forschungsdatenmanagement befasst sind - unter anderem als Sounding Board für das Executive Board agiert und verglich den Launch der EOSC einerseits mit dem Bau eines Schiffs, bei dem zahlreiche Menschen zusammenarbeiten, andererseits aber auch mit einem Marathon, bei dem es um Motivation, Kraft und Ausdauer geht.

Jean-Claude Burgelman, Head of Unit European Commission, Directorate General Research \& Innovation (DG RTD)Unit B2 - Open Science, sprach unter dem Titel "You never walk alone" über die Implementierung der EOSC Roadmap. Die Ziele der EOSC sind: Für europäische Forscherinnen und Forscher eine freie und offene Umgebung zu schaffen, um Daten archivieren, managen, analysieren und wiederverwenden zu können - quer durch alle Disziplinen. Bestehende Infrastrukturen und Lösungen sollen miteinander verbunden und gestärkt werden, Innovation soll weiter ausgebaut werden. Beteiligt sind Geldgeber für Forschung, wissenschaftliche Gesellschaften, Forschungsinfrastrukturen und unter anderem auch Forschungsprojekt-Konsortien. Ein wichtiges Ziel ist die Umsetzung der FAIR-Principles in die Realität. Dabei spielen zahlreiche Komponenten, wie Repositorien, Datenmanagementpläne, Persistent Identifiers, Standards, Lizenzen usw. eine große Rolle. Data Scientists und Data Stewards werden werden in der Zukunft eine große Bedeutung haben. Auf Nachhaltigkeit wird großen Wert gelegt. „Insgesamt liegt noch ein weiter Weg vor uns“, so Burgelman.

\section{Ramping it up}

Nach den beiden einleitenden Präsentationen stellten die fünf bereits existierenden EOSC Projekte ihre Fortschritte und Initiativen zur Implementierung der Basisdienste der EOSC vor.

- EOSC-hub $b^{2}$ als zentraler Kontaktpunkt zum Auffinden, Zugriff und zur Verwendung von Ressourcen der EOSC

- das Projekt OpenAire Advance zur Stärkung der Bewegung zu "Open Science"

- das Projekt FREYA $A^{3}$ zur Erweiterung der Infrastruktur für persistente Identifikatoren

- das Projekt elnfraCentral ${ }^{4}$ zur Definition eines einheitlichen Katalogs für alle Services der EOSC und zur Entwicklung der dazugehörigen zentralen Website 
- RDA Europe 5 als strategischer Partner zur Repräsentation der DatenCommunity in EOSC

Diese und weitere Projekte, alle Beschreibungen, alle Abkürzungen, alle Roadmaps, usw. finden sich in "Prompting an EOSC in practice" dem "Final report and recommendations of the Commission $2^{\text {nd }}$ High Level Expert Group on the European Open Science Cloud (EOSC)". Der Report wurde am Tag nach dem Stakeholders Forum auf dem Launch Event der EOSC an der Uni Wien vorgestellt. Er enthält alle in früheren Dokumenten erarbeiteten Empfehlungen und Überlegungen und kann als Basisdokument der EOSC betrachtet werden.

\section{Governance}

Im Report und auf der Website der $\mathrm{EOSC}^{7}$ sind sechs "Action Lines" zur Implementierung der EOSC beschrieben. Im weiteren Verlauf des Stakeholders Forum wurde in den Sessions für research infrastructures und einfrastructures die bislang erreichte Implementierung der EOSC in den einzelnen Action Lines präsentiert. Mit der Vorstellung der "Governance ${ }^{8 \text { " in }}$ der ersten Session wurde die zukünftige Steuerung der EOSC vorgestellt.

Das Executive Board ist mittlerweile bestellt ${ }^{9}$, die Mitglieder des EOSC Board sind fixiert und die Aufgaben des Stakeholders Forum sind definiert. Die Workgroups und die Definition der "Coordination Support Action Structure (CSA structure)" sind ebenfalls in "Prompting an EOSC in practice" festgelegt. Bis zum Abschluss der Implementierungsphase Ende 2020 besteht die "CSA Structure" aus dem "EOSCsecretariat.eu" ${ }^{10 ", ~ d a s ~ d i e ~ E O S C ~ G o v e r n a n c e ~}$ unterstützen wird.

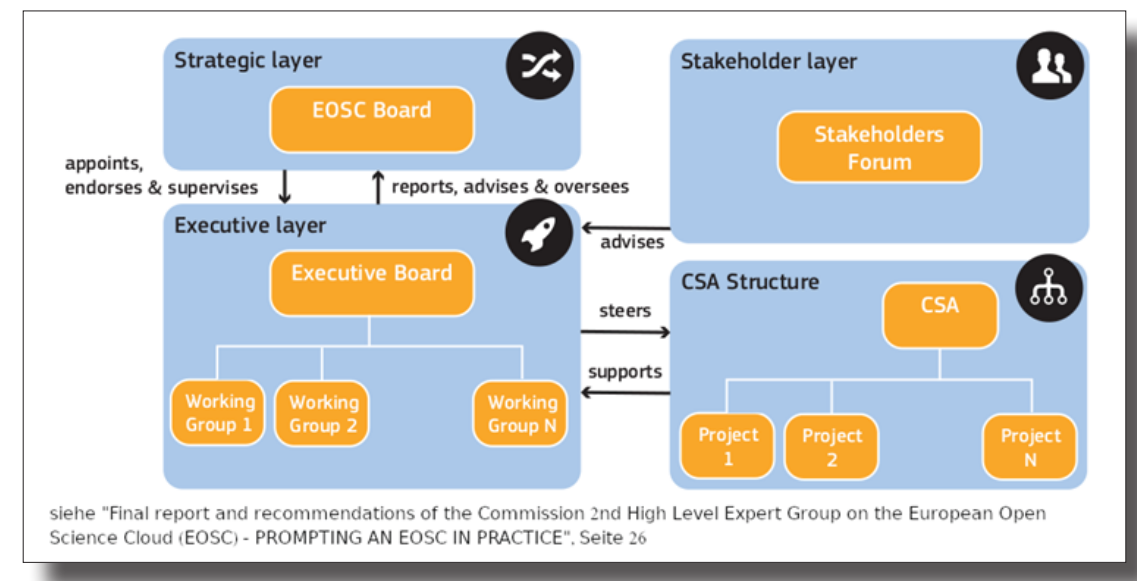




\section{Architektur}

In "Towards EOSC in Practice: Architecture" 11 wurde klargestellt, dass es in der EOSC um "Everything as a Service" geht und nicht nur um Storage oder andere Infrastruktur as a Service (laaS). Es sollen Dienste angeboten werden, die von den Forscherinnen und Forscher in ihrer Arbeit verwendet werden können. Dass die Interoperabilität zwischen den Diensten dabei eine der größten Herausforderungen ist, wurde gleich anschließend in "Interoperability: interfaces, access, and FAIR sharing"12 thematisiert. Als letztes wurde am ersten Tag in "Participation in EOSC: Rules, Obligations, Codes of Conduct and Expectations" über die Regeln zur Teilnahme an der EOSC informiert, die von Service Providern einzuhalten sind.

Die Präsentationen des ersten Tages zeigten, wie viel Arbeit bereits in die EOSC investiert wurde, aber bis zu diesem Zeitpunkt war die am meisten gestellte Frage unter den Teilnehmern die Frage, die auch auf sechs Folien des Closeups als Überschrift stand: "What are we launching?"

\section{Bestehende Forschungsinfrastrukturen und die EOSC}

Der zweite Tag begann mit einer Keynote von Jean-David Malo. "The contribution of the ESFRI infrastructures to the EOSC". In dieser Keynote beschreibt Jean-David, wie die bestehenden ESFRIs zu fünf Clustern zusammengefasst werden, diese Cluster dann jeweils ihre Services nach den Vorgaben von elnfraCentral in den EOSC-hub-Katalog stellen und so die Basis der EOSC bilden. Damit war dann auch klar, „was wir bauen“:

- Mit der EOSC werden im ersten Schritt die Dienstleistungen aller durch die EU geförderten Forschungsinfrastrukturen allgemein zugänglich.

- Weitere Dienste entstehen in den speziellen Arbeitsgruppen die schon vorab gebildet wurden (FREYA,...).

- Kommerzielle Anbieter können in der gleichen Form Dienste wie Forschungsinfrastrukturen anbieten, die von der EU gefördert werden.

Die fünf Cluster sind:

- EOSC-Life ${ }^{13}$ : Dienste von 13 Health and Food Research Infrastructures in die EOSC

- ENVRI-FAIR ${ }^{14}: 13$ Environmental Research Infrastructures definieren Dienste für die EOSC

- ESCAPE: Astronomy \& Particle Physics Cluster

- PaNOSC ${ }^{15}$ : Photon and Neutron Open Science Cloud

- SSHOC ${ }^{16}$ : Social Science und Humanities als Teil der EOSC 
Verantwortliche der fünf Cluster stellten nach der Keynote die bereits erfolgte Anbindung an die EOSC vor. Weitere Informationen finden sich auf den jeweiligen Websites. Erste Projekte der fünf Cluster wurden im EOSCpilot umgesetzt und wurden während der Veranstaltung präsentiert. Sie sind auch auf der EOSCpilot Website als Use Cases ${ }^{17}$ dargestellt.

\section{Policies}

In der anschließenden Panel-Diskussion wurde über Policies diskutiert, die zum effizienten Betrieb, Zugriff und zur Verwendung der EOSC notwendig sind. Das Policy Framework wird von WP3 ${ }^{18}$ des EOSCpilot Projekts erarbeitet und hilft beim Aufbau einer "Ethical, Open, Secure and Cost-effective research environment” in Europa. In der Diskussion wurden hauptsächlich die neun High-Level Empfehlungen des Arbeitspaketes thematisiert, die in der SlideShare Präsentation ${ }^{19}$ zur Diskussion nachzulesen sind.

\section{Skills}

In ihrer Keynote zum Panel "Skills and actions to reward skills acquisition" stellt Françoise Genova klar, dass Data Science und Datenmanagement essentielle Fähigkeiten für Forscherinnen und Forscher sein werden. Sowohl die immer stärker von Datenverarbeitung und Datenanalyse geprägten Forschungsvorhaben, als auch die Vorgaben der Fördergeberlnnen machen es notwendig, dass sich Forscherinnen und Forscher dieses Wissen rasch aneignen können und dafür auch Schulungen angeboten werden. Darüber hinaus muss die Vermittlung der notwendigen Fähigkeiten auch in den Curricula Niederschlag finden. Das Panel war sich einig, dass es ein Anreizsystem für die Forscherinnen und Forscher für die Einhaltung der FAIR-Prinzipien und Forschungsdatenmanagement geben muss. Auch die Fördergeberlnnen sollten Programme zur Belohnung des Erwerbs solcher Fähigkeiten ausrichten.

\section{FAIR-Data}

Im letzten Panel wurden unter dem Titel "How to Implement the FAIR Data Principles?" noch einige sehr wichtige Fragen zu FAIR diskutiert. Einigung bestand in der stärkeren Einbindung der Universitäten in den Prozess. Bei der Frage nach der Sinnhaftigkeit von "Re-Use" entstand eine hitzige Diskussion darüber, wie viele Daten wirklich das Potential zur Wiederverwendbarkeit haben und ob "Re-Use" speziell gefördert werden sollte. 
Dass FAIR für alle Disziplinen gelten sollte und möglichst global eingeführt werden sollte, war anschließend ebenso unbestritten, wie der Ansatz eines möglichst frühen Heranführens der Forscherinnen und Forscher an die FAIR-Prinzipien. Als Streitpunkt erwies sich die letzte Frage nach dem "Wie FAIR sind Daten, die nicht Open-Access sind?" Hier wurde vor allem auf Daten verwiesen, die aus rechtlichen oder lizenzrechtlichen Gründen nicht offen zur Verfügung gestellt werden können. Eine zufriedenstellende Antwort konnte an diesem Tag nicht erarbeitet werden.

\section{Schlussbemerkung}

Die zwei Tage des "EOSCpilot Stakeholder Forum" in Wien haben sichtbar gemacht, wie viel Arbeit und Geld von seiten der EU und der beteiligten Forschungsinfrastrukturen und Institutionen bereits in die EOSC investiert wurden. Ein wesentlicher Teil der notwendigen Infrastruktur, wie zum Beispiel der "EOSC-hub" als zentrales Portal, ist bereits implementiert oder vorbereitet. Einige der teilnehmenden Forschungsinfrastrukturen haben bereits damit begonnen Services in auf EOSC-hub anzubieten.

An anderer Stelle sind noch technische Herausforderungen zu meistern, wie zum Beispiel die Interoperabilität zwischen den Services.

Governance, Policy-Frameworks, Policy-Toolkits und andere Regulatorien sind schon installiert oder werden gerade aufgebaut. Das "EOSCpilot Stakeholder Forum" hat an diesen beiden Tagen letztmalig das "pilot" im Namen getragen. Mit dem Launch der EOSC wird daraus das "EOSC Stakeholder Forum", das eine beratende Funktion für die EOSC Entscheidungsträger haben wird. Bibliotheken und Zentrale Informatikdienste sind zur zukünftigen Teilnahme angehalten, um rechtzeitig informiert zu werden und um sich an den Entwicklungen in der EOSC zu beteiligen.

Zwei Dinge sind auch nach dieser Veranstaltung noch ungeklärt. Da ist zum einen die Finanzierung. Dazu hat es auf der Veranstaltung keine konkreten Aussagen gegeben, aber ein hybrides Modell zwischen Finanzierung durch die Mitgliedstaaten und Weiterverrechnung an die Forscherinnen und Forscher scheint wohl die größten Chancen zu haben. Setzt sich ein solches Finanzierungsmodell durch sind Änderungen der Rahmenbedingungen für forschungsunterstützende Einrichtungen wie Zentrale Informatikdienste und Bibliotheken unausweichlich und müssen gemeinsam mit den Rektoraten diskutiert werden. Und als zweites ist noch immer unklar, welche Vorbereitungen Institutionen treffen müssen, damit ihre Forscherinnen und Forscher auch tatsächlich Dienste aus der EOSC verwenden können. 
Mag. Thomas Haselwanter ORCID: https://orcid.org/0000-0001-9160-0180 Universität Innsbruck, Zentraler Informatikdienst E-Mail: thomas.haselwanter@uibk.ac.at

1 https://eoscpilot.eu/stakeholders-forum/2018-edition/second-eoscpilot-stakeholders-forum-agenda

2 https://www.eosc-hub.eu/

3 https://www.project-freya.eu/en/about/mission

4 http://einfracentral.eu/

5 https://www.rd-alliance.org/rda-europe

6 https://eosc-portal.eu/prompting-eosc-practice

7 https://eosc-portal.eu/about/eosc

8 https://www.slideshare.net/EOSCpiloteu/eosc-governance-sessioneosc-stakeholders-forum-2018?ref=https://www.eoscpilot.eu/content/governance

9 https://eosc-portal.eu/governance

10 https://www.eoscsecretariat.eu/

11 https://www.eoscpilot.eu/content/towards-eosc-practice-architecture

12 https://www.slideshare.net/EOSCpiloteu/eosc-stakeholders-forumenabling-interoperabilityexperience-from-eoscpilot?ref=https://www. eoscpilot.eu/content/interoperability-interfaces-access-and-fair-sharing

13 https://ecrin.org/news/press-release-eosc-life-project-develops-opencollaborative-space-digital-biology-europe

14 http://envri.eu/envri-fair/

15 https://panosc-eu.github.io/

16 https://www.sshopencloud.eu/

17 https://www.eosc-portal.eu/eosc-in-practice/use-cases

18 https://eoscpilot.eu/themes/policy

19 https://www.slideshare.net/EOSCpiloteu/eosc-policy-session-eoscstakeholders-forum-2018?ref=https://eoscpilot.eu/content/policiesopen-ethical-and-secure-eosc 